\title{
Efficacy of Self Directed Learning Program to Improve Technical Institute of Nursing Students Management Competencies
}

\author{
Eman Abdelhamid Shaala ${ }^{1}$, Fouada Mohamed Shabaan ${ }^{2}$ and \\ Karima Ahmed EL-said ${ }^{3}$. \\ ${ }^{1}$ Master in Nursing Service Administration. \\ ${ }^{2}$ Prof. Dr.. of Nursing Administration . Faculty of Nursing - Tanta University . \\ ${ }^{3}$ Assist prof of Nursing Administration, Tanta University .
}

\begin{abstract}
The technical nursing students courses contents give opportunity to apply nursing theory and skills in clinical settings, except nursing administrations course have no clinical application, so they required improving their management competencies. Dimensions of management competencies include four core competencies namely leadership, communication, critical thinking and professionalism. Self-directed learning is the amount of responsibility a student accepts for their own learning. The self-directed learner takes control and accepts the freedom to learn what they consider important for themselves. Readiness for self directed learning is the degree to which the student possesses the attitudes, abilities and personal characteristics necessary for self directed learning. Objective: the aim was to study efficacy of self directed learning program to improve technical institute nursing students management competencies. Setting: The study was conducted at Technical Institute of Nursing at Tanta University. Subject: The study subject (130) technical nursing students include second year students in academic year at 2015-2016. Tool: three tools used including self rating scale of self direct learning readiness, management competencies skills scale and knowledge test on self directed learning. Result: Preprogram about half of nursing students had high level of self directed learning readiness, no one or few of technical nursing students have good levels of all dimensions of management competencies, and all technical nursing student had weak level on domains of self directed learning. Compared to immediate and post 3 months the majority of technical nursing students had high level of total self-directed learning readiness and good skill level of management skill competencies. except communication dimension $(66.9 \%)$ were at good level with significant improvement at $\mathrm{p} 0.05$. Conclusion: technical nursing students at Tanta University Faculty of Nursing showed unsatisfied level of skill for all management competencies dimension, they have great need to attend educational training program about self directed learning to improve their management competencies. Recommendation: conduction pre-employment orientation and periodic educational training program and workshop about management competencies and to use self directed learning.
\end{abstract}

Key words: technical nursing students, management competencies, self directed learning, self directed learning readiness. 


\section{Tanta Scientific Nursing Journal}

\section{INTRODUCTION}

Nurses work in a multifaceted healthcare setting, where they constantly face challenges stemming from the ongoing social and scientific changes inherent in the healthcare field. The responsibility of nursing education is preparing and supporting nurses so that they can successfully adjust and respond to these challenges. Nursing students are expected to be able to fulfil the demands and needs of the community ${ }^{(1)}$. Technical institute of nursing is a two-year program prepares technical nursing students for a defined technical scope of practice ${ }^{(2)}$.

Technical nursing students required to improve their management competencies. Because competency-based education is crucial for safe and efficient nursing services. Nursing student management competency has been defined as the knowledge, skills, ability, and behaviors a student possess to perform tasks correctly and skillfully ${ }^{(3)}$. Lazarte Accreditation management competencies dimensions include four core competencies namely leadership, communication, critical thinking and professionalism. Leadership is vital for all nurses to engage in their roles whether they are involved in direct patient care, work in a consultant position, or serve in positions of formal authority ${ }^{(4)}$.
Interpersonal communication competence is one of the skills that must be cultivated by nursing students for effective achievement of nursing goal in complicated nursing situation, and interaction ${ }^{(5)}$.Critical thinking is the process of actively and skillfully conceptualizing, applying, analyzing, synthesizing, and evaluating information to reach an answer or conclusion. Critical thinking skills are essential to function in today's complex health care environment and to ensure continuing competence for the future ${ }^{(6)}$.

Professionalism is the ability to align personal and organizational conduct with ethical and professional standards that include a responsibility to the patient and community, a service orientation, and a commitment to lifelong learning and improvement $^{(7)}$.

Self-directed learning is the amount of responsibility a student accepts for their own learning. Self directed learning readiness is the degree to which the student possesses the attitudes, abilities and personal characteristics necessary for self directed learning ${ }^{(8)}$. Self directed learning program for technical nursing students management skill competencies and self directed information have tendency to promote their knowledge acquisition and 


\section{Tanta Scientific Nursing Journal}

encourage lifelong learning as well as enables them to improve their management skills competencies and function in their management role safely and proficiently ${ }^{(9)}$.

\section{AIM OF THE STUDY}

To study efficacy of self directed learning program to improve technical institute nursing students management competencies.

\section{RESEARCH HYPOTHESIS}

Technical nursing students readiness to self directed learning and their required management competencies expected to be improved after the self directed learning program.

\section{SUBJECTS AND METHOD}

Study design: Quasi experimental design was used to achieve the aim of study.

Setting: The study was conducted at Technical Institute of Nursing at Tanta University.

Subject: The study subject included second year students in academic year at 2015-2016. The sample size $\mathrm{N}=130$ technical nursing students based on $80 \%$ power analysis and 95\% confidence level of the study (total subject are 385).

Tools: To achieve the aim of the study, three tools were used.

Tool I: Self Rating Scale of Self Direct Learning Readiness: Part (1) this part was involve characteristics of the technical institute nursing students such as age, previous graduation school and training in private hospital.

Part (2)consisted of five broad areas of self-directed learning:

\section{Awareness (personal characteristics)}

- Learning strategies (attitude

- Learning activities (abilities)

- Evaluation.

* Interpersonal skills

* Scoring system

Assess self directed learning readiness measured on five points (1-5) Likert Scale ranging from $1=$ never to $5=$ always .Total scores calculated and represent varying levels of readiness as follows:-High readiness $(>75 \%)=225$ scores, Medium readiness $(60 \%-75 \%)=180-225$, Poor readiness $(<60 \%)=180$ scores

\section{Tool II: Management Competencies}

\section{Skills Scale}

The scale included management skills competencies skills situations for its four dimensions as follows:

1. Leadership dimension

2. Critical thinking dimension

3. Communication dimension

4. Professionalism dimension

Management competencies skills measured on three points Likert Scale ranging from $0=$ not done, $1=$ partial done and $2=$ complete done. Total scores 
calculated and represent varying levels of competency ranging from:Good competent skill $>75 \% 82=$ score,Fair competent skill $60 \%-75 \% \quad 82-66=$ score, Week competent skill $<60 \%=66$ score.

Tool III: knowledge Test on Self Directed Learning included questions about the followings:

a) Self directed learning

b) Competencies and skills to carry out learning plan

c) Managing time related to learning activities

Each question of the test was measured on a score of " 1 " if the answer is "correct" and a score of "zero" if the answer is "wrong". Scores represent varying levels of knowledge as follow: Good knowledge $=(>75 \%)=225$ scores, Fair knowledge $=$ $(60 \%-75 \%)=180-225$ score, Week knowledge $=(<60 \%)=180$ score .

\section{Methods:}

- Official permission to carry out the study was obtained from responsible authorities

- Nursing students conformed consent to participate in the study to obtained (technical nursing students was informed about the privacy of information obtained from them, nature of the study, their rights to withdraw).
- Tools were presented to a jury of 7 experts in nursing administration to check content validity of its items.

- The suitable statistical tests were used for testing tools reliability.

- A pilot study was conducted on $10 \%$ of the total sample to test clarity and applicability of tools and correction was done if necessary.

Research in this study classified into

- Assessment: The self rating scale of self direct learning tool (I), nursing management competencies skills scale (II) and' knowledge test on self directed learning tool (III) were used pre, immediate and after three month for knowledge retest post implementation of the program.

- Plan:Self directed learning program was design and implemented to technical nursing student by researcher based on the assessment data and literature review.

- Objective of the program : At the end of the self directed learning program implementation the technical nursing students will have better readiness to self directed learning as well as their management competencies will be improved.

- Content: The program include ten sessions under ten topics as follows:- 


\section{Tanta Scientific Nursing Journal}

Session (1) Factors affecting management competencies improvement.

Session (2) Dimensions of management competencies.

Session (3) Instructional methods to improve self directed learning readiness.

Session (4) Self direction in learning.

Session (5) Competencies and skills to carry out learning plan.

Session (6) Managing time related to learning activities.

Session $(7,8,9,10)$ Examples of clinical situation to train technical nursing students how to implement self directed learning process on leadership, communication, critical thinking, and professionalism respectively.

- Implementation of the program:Technical nursing student divided into ten groups. The program (10) sessions every session 2 hours for every group. The program (20 hours) conducted for technical nursing student, at their technical nursing institute.

- Evaluation of the program: Pre, immediate and after three month post implementation of the program. Evaluation done on technical nursing students self directed learning knowledge, nursing management competencies as well as their self directed learning readiness.
- Learning strategies: Appropriate learning strategies used as power point, assignment, group discussion, situation, slides, videos, role-plays.......etc.

\section{RESULTS}

Table (1) Shows characteristics of the technical nursing students. The age of technical nursing student were ranged from 19-29 years with mean age $20.02 \pm 1.403$. High percent $(92.3 \%)$ of technical nursing student were in the age group $\leq 20$ years and the rest $7.7 \%$ were in the age group $>20$ years. Majority (96.9\%) of technical nursing student graduated from General secondary school, while minority $(3.1 \%)$ graduated from Secondary technical school. Technical nursing student $73.1 \%$ did not have any previous training and $26.9 \%$ had previous training with training mean period $6.83 \pm 12.906$ month.

Figure (1) Show nursing students' level of total self directed learning readiness. Pre program about half of nursing students had high level of self directed learning readiness compared to immediate and post 3 months the majority had high level of total self directed learning readiness.

Table (2) Represents technical nursing students levels and means of self directed learning readiness domains. The table shows statistical significant difference 


\section{Tanta Scientific Nursing Journal}

between nursing students levels and means for all self rating domains at $\mathrm{P}=0.00$. Preprogram range $5.4 \%$ - $3.1 \%$ had poor level while $53.8-69.2 \%$ had high level regarding awareness, learning strategies, evaluation, interpersonal skills domains of self rating. All technical nursing students had high levels of interpersonal skills domains of self rating immediate and post 3 months of program. While majority of technical nursing students range 99.2\%$96.9 \%$ had high level of learning strategies, evaluation, awareness and learning activities domains of self rating respectively immediate and post 3 months of program. Pre program mean score was 48.25 $\pm 5.187,48.88 \pm 5.361,44.74 \pm 6.021$,

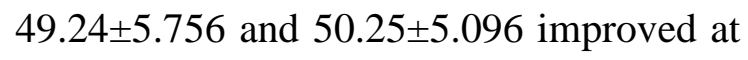
both immediate and post 3 months to be $56.78 \pm 2.942 ， 55.58 \pm 3.647 ， 55.58 \pm 3.647$, $57.63 \pm 2.989$ and $58.11 \pm 2.051$ respectively regarding awareness, learning strategies, learning activities, evaluation, and interpersonal skills domains of self rating.

Table (3) Represents total score of selfdirected learning readiness domains pre and post program according to student characteristics. The table illustrates statistical significant correlation between total learning activities of self directed learning readiness general and technical previous graduation schools preprogram implementation at $\mathrm{P}<0.01$. Moreover statistical significant correlation found between total score of self-rating and previous graduation school preprogram implementation at $\mathrm{P}<0.05$.

Figure (2) Represent technical nursing students level of total management competencies skills pre, immediate and 3 month post program. No one of technical nursing students have good level of management competencies skills pre program, changed to be all of them have good level of management competencies skills immediate and 3 months post program.

Table (4) Represent technical nursing students levels and mean of management competencies skills dimensions pre, post and 3month post program. Statistical significant difference found between students levels and means of management competencies skills dimensions pre, immediate, post 3 months program implementation at $\mathrm{P}<$ 0.05. Preprogram no one or few of technical nursing students have good levels of all dimensions of management competencies. But immediate and 3months post program all technical nursing students were at good level except communication dimension was (66.9\%). Preprogram students mean 


\section{Tanta Scientific Nursing Journal}

scores were $4.92 \pm 1.29,1.89 \pm 1.05$ while immediate, and post 3months significantly improved to reach $11.00 \pm 0.00, \quad 4.00 \pm 0.00 \quad$ regarding leadership and critical thinking dimensions respectively.

Table (5)Technical nursing students total score of management competencies skills dimension pre and post program according to their characteristics .The table illustrates statistical significant correlation between students total management competencies skills, communication, and professionalism with their age post program at $\mathrm{P}<0.05$.

Figure (3) Represent technical nursing students total level of knowledge on self directed learning throughout pre, post, 3month post program. Preprogram implementation all technical nursing students had weak level of knowledge on self directed learning while immediate post program all students had good level, slightly decreased 3months post program to be majority had good level.

Table (6)Represents technical nursing students levels of knowledge on domains of self directed learning pre, immediate, 3month post program. Statistical significant improvement found between level and mean of total knowledge of domains for self directed learning pre, immediate, post 3 months program at $\mathrm{P}$ $<0.05$. Preprogram all technical nursing student had weak level on all domains of self directed learning and changed to be good level immediate and post 3month of program. Also preprogram technical nursing students mean scores of knowledge was $\quad 7.87 \pm 0.438$, $12.06 \pm 0.878, \quad 4.85 \pm 0.590 \quad$ while immediate, post 3 months program were significantly improved to reach $20.00 \pm 0.00, \quad 27.00 \pm 0.00,13.00 \pm 0.00$ regarding to self directed learning, competencies and skills to carry out learning plan, and managing time to learning activities domains respectively.

Table (7) Represent correlation between the all domains of self-rating scale, management competencies skills and knowledge on self directed learning among the studied technical nursing students pre and post program. Regarding management competencies skill, this table shows significant correlations between leadership and, learning activities and interpersonal skills at $\mathrm{P}<0.05$ pre program. In addition, positive correlation were observed regarding communication and learning activities and interpersonal skills where at $\mathrm{P}<0.05$ pre program. On the other hand, a negative and significant correlation was observed in relation to professionalism and 


\section{Tanta Scientific Nursing Journal}

interpersonal skills for $\mathrm{r}=-0.202$ and $\mathrm{P}=$ 0.021 . In relation to knowledge on self directed learning domain, this table reported positive and significant correlations between self-directed learning, learning strategies and interpersonal skills at $\mathrm{P}<0.05$ pre program. Also, positive correlations were found between competencies and skills to carry out learning plan and awareness, learning strategies preprogram at $\mathrm{P}<0.05$. Also, positive correlations were observed pre and post program between competencies and interpersonal skills at $\mathrm{P}<0.05$.On the other hand, negative and significant correlations were observed between managing time related to learning activities, awareness and interpersonal skills at $\mathrm{P}<0.05$ and $\mathrm{r}=-0.253$ and -0.178 respectively. 
Table (1): Characteristics of the technical nursing students.

\begin{tabular}{|c|c|c|}
\hline \multirow{2}{*}{ Characteristics } & \multicolumn{2}{|c|}{$\begin{array}{l}\text { Technical nursing students } \\
\qquad(n=130)\end{array}$} \\
\hline & $\mathbf{N}$ & $\%$ \\
\hline $\begin{array}{l}\text { Age }(\text { in years }) \\
-\quad \leq 20 \text { years } \\
->20 \text { years }\end{array}$ & $\begin{array}{c}120 \\
10\end{array}$ & $\begin{array}{c}92.3 \\
7.7\end{array}$ \\
\hline $\begin{array}{c}\text { Range } \\
\text { Mean } \pm \text { SD }\end{array}$ & \multicolumn{2}{|c|}{$\begin{array}{c}(19-29) \\
20.02 \pm 1.403\end{array}$} \\
\hline $\begin{array}{l}\text { Previous graduation school } \\
- \text { General secondary school } \\
-\quad \text { Secondary technical school }\end{array}$ & $\begin{array}{c}126 \\
4\end{array}$ & $\begin{array}{c}96.9 \\
3.1\end{array}$ \\
\hline $\begin{array}{l}\text { Previous training in private hospital } \\
- \text { No } \\
- \text { Yes }\end{array}$ & $\begin{array}{l}95 \\
35\end{array}$ & $\begin{array}{l}73.1 \\
26.9\end{array}$ \\
\hline $\begin{array}{c}\text { Period of that training (in months) } \\
\text { Range } \\
\text { Mean } \pm \text { SD }\end{array}$ & & $\begin{array}{l}\text { 2) } \\
2.906\end{array}$ \\
\hline
\end{tabular}

霖 Poor Medium $\mathrm{E}$ High

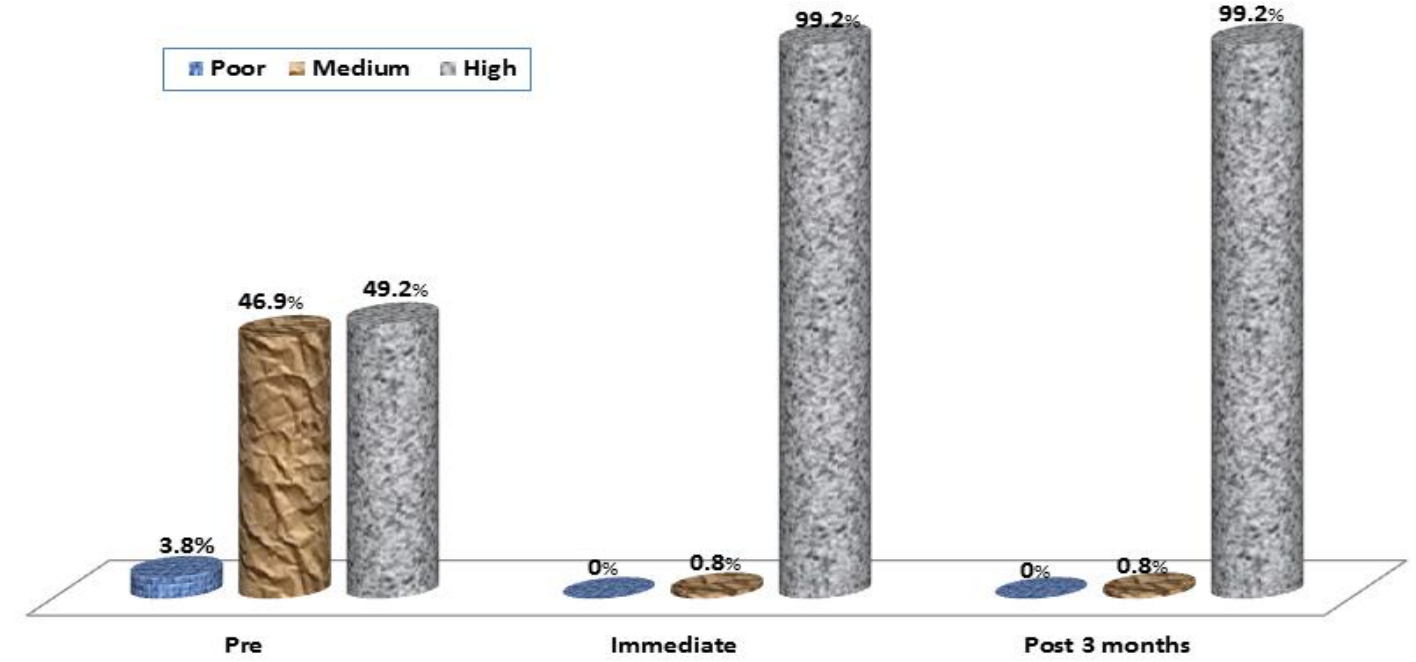

Figure (1): Nursing students level of total self directed learning readiness pre, immediate, 3month post program. 
Table (2): Technical nursing students levels and means of self directed learning readiness domains.

\begin{tabular}{|c|c|c|c|c|c|c|c|}
\hline \multirow{3}{*}{$\begin{array}{l}\text { Levels of self rating } \\
\text { scale domains }\end{array}$} & \multicolumn{6}{|c|}{ Technical nursing students $(n=130)$} & \multirow{3}{*}{$\begin{array}{l}\chi^{2} \\
\mathbf{P}\end{array}$} \\
\hline & \multicolumn{2}{|c|}{ Pre } & \multicolumn{2}{|c|}{ Immediate } & \multicolumn{2}{|c|}{$\begin{array}{c}\text { Post } 3 \\
\text { Months }\end{array}$} & \\
\hline & $\mathbf{N}$ & $\%$ & $\mathbf{N}$ & $\%$ & $\mathbf{N}$ & $\%$ & \\
\hline \begin{tabular}{ll}
\multicolumn{2}{l}{ Total awareness } \\
- & Poor \\
- & Medium \\
- & High
\end{tabular} & $\begin{array}{c}7 \\
53 \\
70\end{array}$ & $\begin{array}{c}5.4 \\
40.8 \\
53.8\end{array}$ & $\begin{array}{c}0 \\
3 \\
127\end{array}$ & $\begin{array}{c}0.0 \\
2.3 \\
97.7\end{array}$ & $\begin{array}{c}0 \\
3 \\
127\end{array}$ & $\begin{array}{c}0.0 \\
2.3 \\
97.7\end{array}$ & $\begin{array}{c}118.80 \\
0.00 *\end{array}$ \\
\hline $\begin{array}{c}\text { Range } \\
\text { Mean } \pm \text { SD }\end{array}$ & \multicolumn{2}{|c|}{$\begin{array}{c}(30-60) \\
48.25 \pm 5.187\end{array}$} & \multicolumn{2}{|c|}{$\begin{array}{c}(45-60) \\
56.78 \pm 2.942\end{array}$} & \multicolumn{2}{|c|}{$\begin{array}{c}(45-60) \\
56.74 \pm 2.932\end{array}$} & $\begin{array}{c}\mathbf{F}=\mathbf{2 1 2 . 9 0} \\
\mathbf{P}=0.00 *\end{array}$ \\
\hline $\begin{array}{ll}\text { Total learning } & \text { strategies } \\
- & \text { Poor } \\
- & \text { Medium } \\
- & \text { High }\end{array}$ & $\begin{array}{l}10 \\
52 \\
68\end{array}$ & $\begin{array}{c}7.7 \\
40.0 \\
52.3\end{array}$ & $\begin{array}{c}0 \\
1 \\
129\end{array}$ & $\begin{array}{c}0.0 \\
0.8 \\
99.2\end{array}$ & $\begin{array}{c}0 \\
1 \\
129\end{array}$ & $\begin{array}{c}0.0 \\
0.8 \\
99.2\end{array}$ & $\begin{array}{c}139.16 \\
0.00 *\end{array}$ \\
\hline $\begin{array}{c}\text { Range } \\
\text { Mean } \pm \text { SD }\end{array}$ & \multicolumn{2}{|c|}{$\begin{array}{c}(35-60) \\
48.88 \pm 5.361\end{array}$} & \multicolumn{2}{|c|}{$\begin{array}{c}(47-60) \\
57.25 \pm 2.774\end{array}$} & \multicolumn{2}{|c|}{$\begin{array}{c}(47-60) \\
57.25 \pm 2.774\end{array}$} & $\begin{array}{c}F=206.70 \\
P=0.00 *\end{array}$ \\
\hline \begin{tabular}{ll}
\multicolumn{2}{l}{ Total learning activities } \\
- & Poor \\
- & Medium \\
- & High
\end{tabular} & $\begin{array}{l}31 \\
64 \\
35\end{array}$ & $\begin{array}{l}23.8 \\
49.2 \\
26.9\end{array}$ & $\begin{array}{c}0 \\
4 \\
126\end{array}$ & $\begin{array}{c}0.0 \\
3.1 \\
96.9\end{array}$ & $\begin{array}{c}0 \\
4 \\
126\end{array}$ & $\begin{array}{c}0.0 \\
3.1 \\
96.9\end{array}$ & $\begin{array}{c}219.71 \\
0.00 *\end{array}$ \\
\hline $\begin{array}{c}\text { Range } \\
\text { Mean } \pm \text { SD }\end{array}$ & \multicolumn{2}{|c|}{$\begin{array}{c}(30-60) \\
44.74 \pm 6.021\end{array}$} & \multicolumn{2}{|c|}{$\begin{array}{c}(42-60) \\
55.58 \pm 3.647\end{array}$} & \multicolumn{2}{|c|}{$\begin{array}{c}(42-60) \\
55.58 \pm 3.647\end{array}$} & $\begin{array}{c}\mathbf{F}=243.31 \\
\mathbf{P}=0.00 *\end{array}$ \\
\hline \begin{tabular}{ll}
\multicolumn{2}{l}{ Total evaluation } \\
- & Poor \\
- & Medium \\
- & High \\
\end{tabular} & $\begin{array}{c}8 \\
52 \\
70\end{array}$ & $\begin{array}{c}6.2 \\
40.0 \\
53.8\end{array}$ & $\begin{array}{c}0 \\
1 \\
129\end{array}$ & $\begin{array}{c}0.0 \\
0.8 \\
99.2\end{array}$ & $\begin{array}{c}0 \\
1 \\
129\end{array}$ & $\begin{array}{c}0.0 \\
0.8 \\
99.2\end{array}$ & $\begin{array}{c}133.56 \\
0.00 *\end{array}$ \\
\hline $\begin{array}{c}\text { Range } \\
\text { Mean } \pm \text { SD }\end{array}$ & \multicolumn{2}{|c|}{$\begin{array}{c}(30-60) \\
49.24 \pm 5.756\end{array}$} & \multicolumn{2}{|c|}{$\begin{array}{c}(42-60) \\
57.63 \pm 2.989\end{array}$} & \multicolumn{2}{|c|}{$\begin{array}{c}(42-60) \\
57.63 \pm 2.989\end{array}$} & $\begin{array}{c}F=179.55 \\
P=0.00 *\end{array}$ \\
\hline $\begin{array}{ll}\text { Total interpersonal skills } \\
- & \text { Poor } \\
- & \text { Medium } \\
- & \text { High }\end{array}$ & $\begin{array}{c}4 \\
36 \\
90\end{array}$ & $\begin{array}{c}3.1 \\
27.7 \\
69.2\end{array}$ & $\begin{array}{c}0 \\
0 \\
130\end{array}$ & $\begin{array}{c}0.0 \\
0.0 \\
100.0\end{array}$ & $\begin{array}{c}0 \\
0 \\
130\end{array}$ & $\begin{array}{c}0.0 \\
0.0 \\
100.0\end{array}$ & $\begin{array}{l}89.14 \\
0.00 *\end{array}$ \\
\hline $\begin{array}{c}\text { Range } \\
\text { Mean } \pm \text { SD }\end{array}$ & \multicolumn{2}{|c|}{$\begin{array}{c}(32-60) \\
50.25 \pm 5.096\end{array}$} & \multicolumn{2}{|c|}{$\begin{array}{c}(49-60) \\
58.11 \pm 2.051\end{array}$} & \multicolumn{2}{|c|}{$\begin{array}{c}(49-60) \\
58.11 \pm 2.051\end{array}$} & $\begin{array}{c}F=233.69 \\
P=0.00 *\end{array}$ \\
\hline
\end{tabular}

* Significant at $\mathrm{P}<0.05$ 


\section{Tanta Scientific Nursing Journal}

Table (3): Total score of self-directed learning readiness domains pre and post program according to student characteristics.

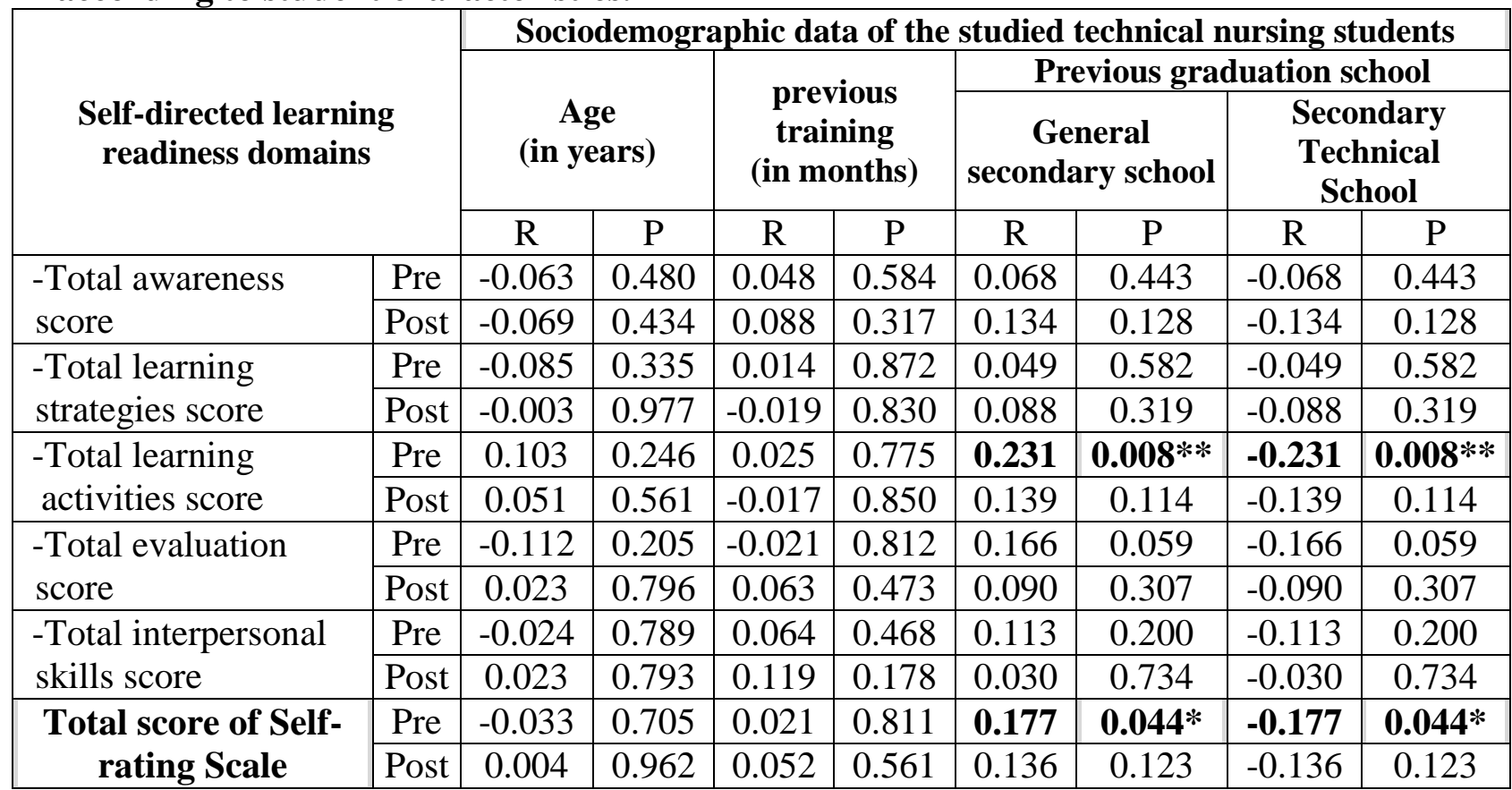

* Significant at $\mathrm{P}<0.05 * *$ Highly significant at $\mathrm{P}<0.01$

Table (4): Technical nursing students levels and mean of management competencies skills dimensions.

\begin{tabular}{|c|c|c|c|c|c|c|c|}
\hline \multirow{3}{*}{$\begin{array}{l}\text { Levels of Management Competencies Skills } \\
\text { Scale dimensions }\end{array}$} & \multicolumn{6}{|c|}{ Technical nursing students $(\mathrm{N}=130)$} & \multirow{3}{*}{$\stackrel{\chi^{2}}{\mathbf{P}}$} \\
\hline & \multicolumn{2}{|c|}{ Pre } & \multicolumn{2}{|c|}{ Immediate } & \multicolumn{2}{|c|}{$\begin{array}{c}\text { Post 3 } \\
\text { months }\end{array}$} & \\
\hline & $\mathbf{N}$ & $\%$ & $\mathbf{N}$ & $\%$ & $\mathbf{N}$ & $\%$ & \\
\hline $\begin{array}{l}\text { A. Total Leadership } \\
\text { Weak } \\
\text { - Fair } \\
\text { Good }\end{array}$ & $\begin{array}{c}113 \\
17 \\
0\end{array}$ & $\begin{array}{c}86.9 \\
13.1 \\
0.0\end{array}$ & \begin{tabular}{|c|}
0 \\
0 \\
130 \\
\end{tabular} & $\begin{array}{c}0.0 \\
0.0 \\
100.0\end{array}$ & $\begin{array}{c}0 \\
0 \\
0 \\
130\end{array}$ & $\begin{array}{c} \\
0.0 \\
0.0 \\
100.0\end{array}$ & $\begin{array}{c}390.00 \\
0.00 *\end{array}$ \\
\hline$\underset{\text { Mean } \pm \text { SD }}{\text { Range }}$ & \multicolumn{2}{|c|}{$\begin{array}{c}(2-8) \\
4.92 \pm 1.29\end{array}$} & \multicolumn{2}{|c|}{$\begin{array}{c}(11-11) \\
11.00 \pm 0.00\end{array}$} & \multicolumn{2}{|c|}{$\begin{array}{c}(11-11) \\
11.00 \pm 0.00\end{array}$} & $\begin{array}{c}F=2873.47 \\
P=0.00 *\end{array}$ \\
\hline $\begin{array}{l}\text { B. } \text { Total critical thinking } \\
\text { Weak } \\
\text { - Fair } \\
\text { - Good }\end{array}$ & $\begin{array}{c}93 \\
30 \\
7\end{array}$ & $\begin{array}{l}71.5 \\
23.1 \\
5.4\end{array}$ & $\begin{array}{c}0 \\
0 \\
130\end{array}$ & $\begin{array}{c}0.0 \\
0.0 \\
100.0\end{array}$ & $\begin{array}{c}0 \\
0 \\
130\end{array}$ & $\begin{array}{c}0.0 \\
0.0 \\
100.0\end{array}$ & $\begin{array}{c}359.33 \\
0.00^{*}\end{array}$ \\
\hline $\begin{array}{c}\text { Range } \\
\text { Mean } \pm \text { SD }\end{array}$ & \multicolumn{2}{|c|}{$\begin{array}{c}(0-4) \\
1.89 \pm 1.05\end{array}$} & \multicolumn{2}{|c|}{$\begin{array}{c}(4-4) \\
4.00 \pm 0.00\end{array}$} & \multicolumn{2}{|c|}{$\begin{array}{c}(4-4) \\
4.00 \pm 0.00\end{array}$} & $\begin{array}{c}F=522.83 \\
P=0.00^{*}\end{array}$ \\
\hline $\begin{array}{l}\text { C. } \text { Total Communication } \\
\text { Weak } \\
\text { Fair } \\
\text { Good }\end{array}$ & $\begin{array}{c}128 \\
2 \\
0\end{array}$ & $\begin{array}{l}98.5 \\
1.5 \\
0.0\end{array}$ & \begin{tabular}{|c|}
0 \\
0 \\
130 \\
\end{tabular} & $\begin{array}{c}0.0 \\
0.0 \\
100.0\end{array}$ & $\begin{array}{l}7 \\
36 \\
87\end{array}$ & $\begin{array}{r}5.4 \\
27.7 \\
66.9\end{array}$ & $\begin{array}{r}416.09 \\
0.00 *\end{array}$ \\
\hline $\begin{array}{c}\text { Range } \\
\text { Mean } \pm \text { SD }\end{array}$ & \multicolumn{2}{|c|}{$\begin{array}{c}(0-4) \\
1.50 \pm 0.93\end{array}$} & \multicolumn{2}{|c|}{$\begin{array}{c}(5-5) \\
5.00 \pm 0.00\end{array}$} & \multicolumn{2}{|c|}{$\begin{array}{c}(3-5) \\
4.62 \pm 0.59\end{array}$} & $\begin{array}{c}F=1178.48 \\
P=0.00^{*}\end{array}$ \\
\hline $\begin{array}{l}\text { D. Total professionalism } \\
\text { - Weak } \\
\text { Fair }\end{array}$ & $\begin{array}{c}129 \\
1\end{array}$ & $\begin{array}{c}99.2 \\
0.8\end{array}$ & $\begin{array}{c}0 \\
130\end{array}$ & $\begin{array}{c}0.0 \\
100.0\end{array}$ & $\begin{array}{c}3 \\
127\end{array}$ & $\begin{array}{c}2.3 \\
97.7\end{array}$ & $\begin{array}{c}372.48 \\
0.00 *\end{array}$ \\
\hline $\begin{array}{c}\text { Range } \\
\text { Mean } \pm \text { SD }\end{array}$ & \multicolumn{2}{|c|}{$\begin{array}{c}(0-5) \\
1.69 \pm 0.97\end{array}$} & \multicolumn{2}{|c|}{\begin{tabular}{|c|}
$(6-6)$ \\
$6.00 \pm 0.00$ \\
\end{tabular}} & \multicolumn{2}{|c|}{$\begin{array}{c}(4-6) \\
5.75 \pm 0.49\end{array}$} & $\begin{array}{c}F=1929.75 \\
P=0.00 *\end{array}$ \\
\hline
\end{tabular}




\section{Tanta Scientific Nursing Journal}

Table (5): Technical students total management competencies skills dimension, pre and post program according to their characteristics

\begin{tabular}{|c|c|c|c|c|c|c|c|c|c|}
\hline \multirow{4}{*}{\multicolumn{2}{|c|}{$\begin{array}{l}\text { Total scores of management } \\
\text { competencies skills } \\
\text { dimension }\end{array}$}} & \multicolumn{8}{|c|}{$\begin{array}{c}\text { Sociodemographic data of technical } \\
\text { nursing students }\end{array}$} \\
\hline & & \multirow{2}{*}{\multicolumn{2}{|c|}{$\begin{array}{c}\text { Age } \\
\text { (in years) }\end{array}$}} & \multirow{2}{*}{\multicolumn{2}{|c|}{$\begin{array}{c}\text { Private } \\
\text { training } \\
\text { (in months) }\end{array}$}} & \multicolumn{4}{|c|}{ Previous graduation school } \\
\hline & & & & & & \multicolumn{2}{|c|}{$\begin{array}{c}\text { General } \\
\text { secondary } \\
\text { school }\end{array}$} & \multicolumn{2}{|c|}{$\begin{array}{c}\text { Secondary } \\
\text { Technical } \\
\text { School }\end{array}$} \\
\hline & & $\mathrm{R}$ & $\mathrm{P}$ & $\mathrm{r}$ & $\mathrm{P}$ & $\mathrm{R}$ & $\mathrm{P}$ & $\mathrm{R}$ & $\mathrm{P}$ \\
\hline \multirow{2}{*}{ 1. Total leadership } & Pre & 0.135 & 0.127 & -0.003 & 0.975 & -0.029 & 0.741 & 0.029 & 0.741 \\
\hline & Post & - & 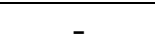 & - & - & - & - & - & - \\
\hline \multirow{2}{*}{ 2. Total communication } & Pre & 0.132 & 0.135 & -0.149 & 0.092 & 0.098 & 0.266 & -0.098 & 0.266 \\
\hline & Post & 0.174 & 0.048* & -0.093 & 0.293 & -0.124 & 0.160 & 0.124 & 0.160 \\
\hline \multirow{2}{*}{ 3. Total critical thinking } & Pre & 0.043 & 0.625 & 0.066 & 0.454 & -0.066 & 0.454 & 0.066 & 0.454 \\
\hline & Post & - & - & - & - & - & - & - & - \\
\hline \multirow{2}{*}{ 4. Total professionalism } & Pre & 0.027 & 0.764 & -0.016 & 0.858 & 0.035 & 0.692 & -0.035 & 0.692 \\
\hline & Post & 0.185 & $\mathbf{0 . 0 3 5} *$ & 0.112 & 0.203 & -0.097 & 0.271 & 0.097 & 0.271 \\
\hline \multirow{2}{*}{$\begin{array}{c}\text { Total score of } \\
\text { management } \\
\text { Competencies skills }\end{array}$} & Pre & 0.173 & 0.049* & -0.090 & 0.310 & 0.018 & 0.838 & -0.018 & 0.838 \\
\hline & Post & 0.233 & $0.008 * *$ & 0.013 & 0.882 & -0.156 & 0.077 & 0.156 & 0.077 \\
\hline
\end{tabular}

* Significant at $\mathrm{P}<0.05$

** Highly significant at $\mathrm{P}<0.01$

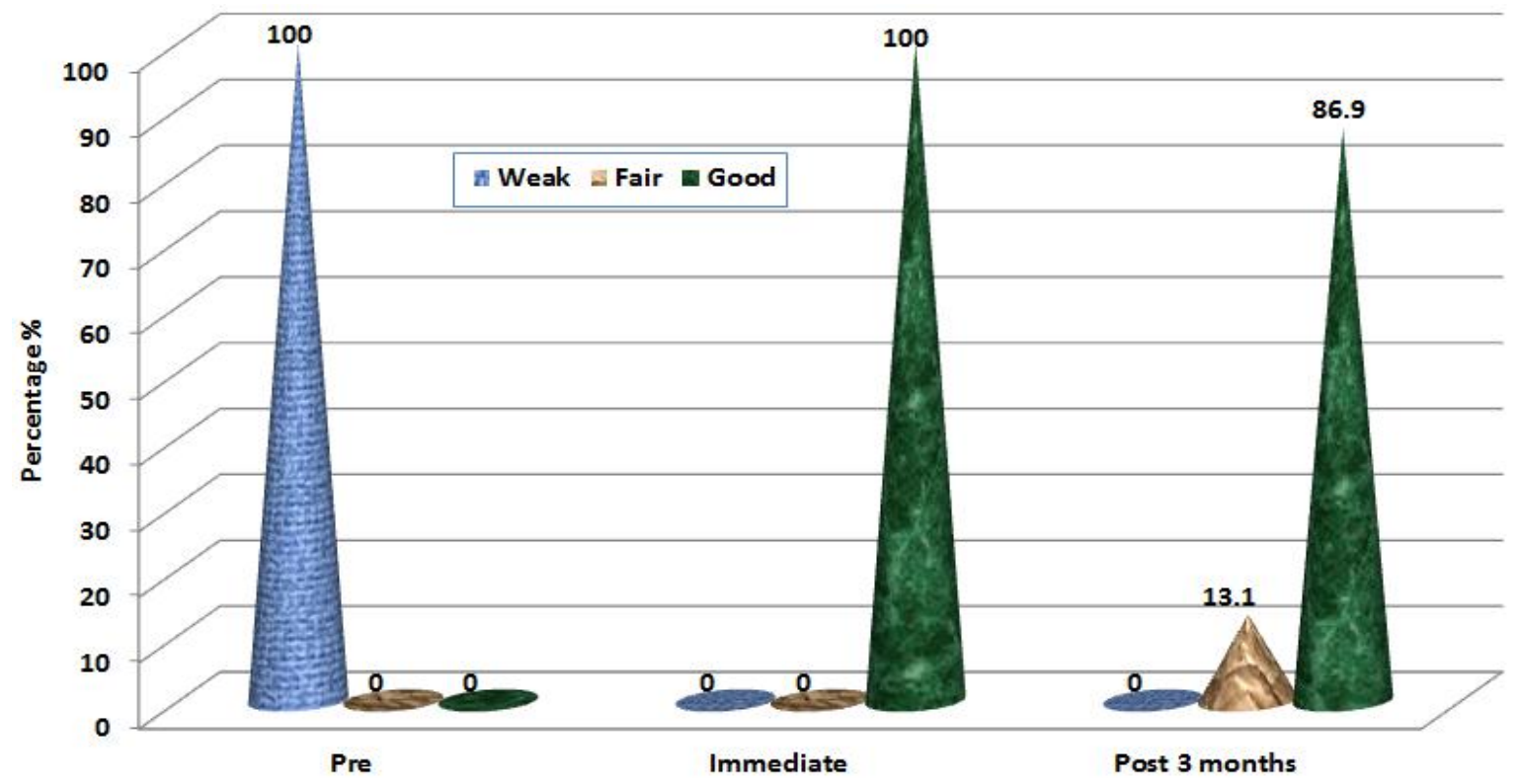

Figure (3) levels of knowledge on self directed learning throughout pre, post, post 3month program. 
Table (6): Technical nursing students levels of knowledge on domains self directed learning pre, immediate, 3 month post program.

\begin{tabular}{|c|c|c|c|c|c|c|c|}
\hline \multirow{3}{*}{$\begin{array}{l}\text { Levels of knowledge } \\
\text { domains on Self } \\
\text { Directed Learning }\end{array}$} & \multicolumn{6}{|c|}{$\begin{array}{l}\text { Technical nursing students } \\
\qquad(\mathrm{N}=130)\end{array}$} & \multirow{3}{*}{$\begin{array}{l}\chi^{2} \\
\mathbf{P}\end{array}$} \\
\hline & \multicolumn{2}{|c|}{ Pre } & \multicolumn{2}{|c|}{$\underset{\mathrm{e}}{\text { Immediat }}$} & \multicolumn{2}{|c|}{$\begin{array}{l}\text { Post } 3 \\
\text { months }\end{array}$} & \\
\hline & $\mathbf{N}$ & $\%$ & $\mathbf{N}$ & $\%$ & $\mathbf{N}$ & $\%$ & \\
\hline $\begin{array}{l}\text { - Self directed learning } \\
\text { - Weak } \\
\text { - Fair } \\
\text { - Good }\end{array}$ & $\begin{array}{c}130 \\
0 \\
0\end{array}$ & $\begin{array}{c}100.0 \\
0.0 \\
0.0\end{array}$ & $\begin{array}{c}0 \\
0 \\
130\end{array}$ & $\begin{array}{c}0.0 \\
0.0 \\
100.0\end{array}$ & $\begin{array}{c}0 \\
40 \\
90 \\
\end{array}$ & $\begin{array}{c}0.0 \\
30.8 \\
69.2 \\
\end{array}$ & $\begin{array}{c}460.91 \\
0.00 *\end{array}$ \\
\hline $\begin{array}{c}\text { Range } \\
\text { Mean } \pm \text { SD }\end{array}$ & \multicolumn{2}{|c|}{$\begin{array}{c}(5-9) \\
7.87 \pm 0.438\end{array}$} & \multicolumn{2}{|c|}{$\begin{array}{c}(20-20) \\
20.00 \pm 0.0 \\
0\end{array}$} & \multicolumn{2}{|c|}{$\begin{array}{c}(13-19) \\
16.25 \pm 1.30 \\
2\end{array}$} & $\begin{array}{c}F=7974.7 \\
P=0.00 *\end{array}$ \\
\hline $\begin{array}{l}\text { - Competencies and skills to } \\
\text { carry out learning plan } \\
\text { - Weak } \\
\text { - Fair } \\
\text { - Good }\end{array}$ & $\begin{array}{c}130 \\
0 \\
0\end{array}$ & $\begin{array}{c}100.0 \\
0.0 \\
0.0\end{array}$ & $\begin{array}{c}0 \\
0 \\
130\end{array}$ & $\begin{array}{c}0.0 \\
0.0 \\
100.0\end{array}$ & $\begin{array}{c}0 \\
38 \\
92\end{array}$ & $\begin{array}{c}0.0 \\
29.2 \\
70.8\end{array}$ & $\begin{array}{c}456.76 \\
0.00 *\end{array}$ \\
\hline $\begin{array}{c}\text { Range } \\
\text { Mean } \pm \text { SD }\end{array}$ & \multicolumn{2}{|c|}{\begin{tabular}{|c|}
$(9-13)$ \\
$12.06 \pm 0.87$ \\
8
\end{tabular}} & \multicolumn{2}{|c|}{$\begin{array}{c}(27-27) \\
27.00 \pm 0.0 \\
0\end{array}$} & \multicolumn{2}{|c|}{\begin{tabular}{c|}
$(18-26)$ \\
$21.42 \pm 1.66$ \\
1
\end{tabular}} & $\begin{array}{c}\mathrm{F}=6297.4 \\
\mathbf{P}=0.00 *\end{array}$ \\
\hline $\begin{array}{l}\text { - Managing time related to } \\
\text { learning activities } \\
\text { - Weak } \\
\text { - Fair } \\
\text { - Good }\end{array}$ & $\begin{array}{c}130 \\
0 \\
0\end{array}$ & $\begin{array}{c}100.0 \\
0.0 \\
0.0\end{array}$ & $\begin{array}{c}0 \\
0 \\
130\end{array}$ & $\begin{array}{c}0.0 \\
0.0 \\
100.0 \\
\end{array}$ & $\begin{array}{c}1 \\
14 \\
115 \\
\end{array}$ & $\begin{array}{c}0.7 \\
10.8 \\
88.5\end{array}$ & $\begin{array}{c}407.92 \\
0.00 *\end{array}$ \\
\hline $\begin{array}{c}\text { Range } \\
\text { Mean } \pm \text { SD }\end{array}$ & \multicolumn{2}{|c|}{$\begin{array}{c}(4-6) \\
4.85 \pm 0.590\end{array}$} & \multicolumn{2}{|c|}{\begin{tabular}{|c|}
$(13-13)$ \\
$13.00 \pm 0.0$ \\
0
\end{tabular}} & \multicolumn{2}{|c|}{\begin{tabular}{c|}
$(7-13)$ \\
$10.55 \pm 0.97$ \\
3 \\
\end{tabular}} & $\begin{array}{c}F=5267.2 \\
P=0.00 *\end{array}$ \\
\hline
\end{tabular}

* Significant at $\mathrm{P}<0.05$ 


\section{Tanta Scientific Nursing Journal}

Table (7): Correlation between the all domains of self-rating scale, management competencies skills and knowledge on self directed learning among the studied technical nursing students pre and post program.

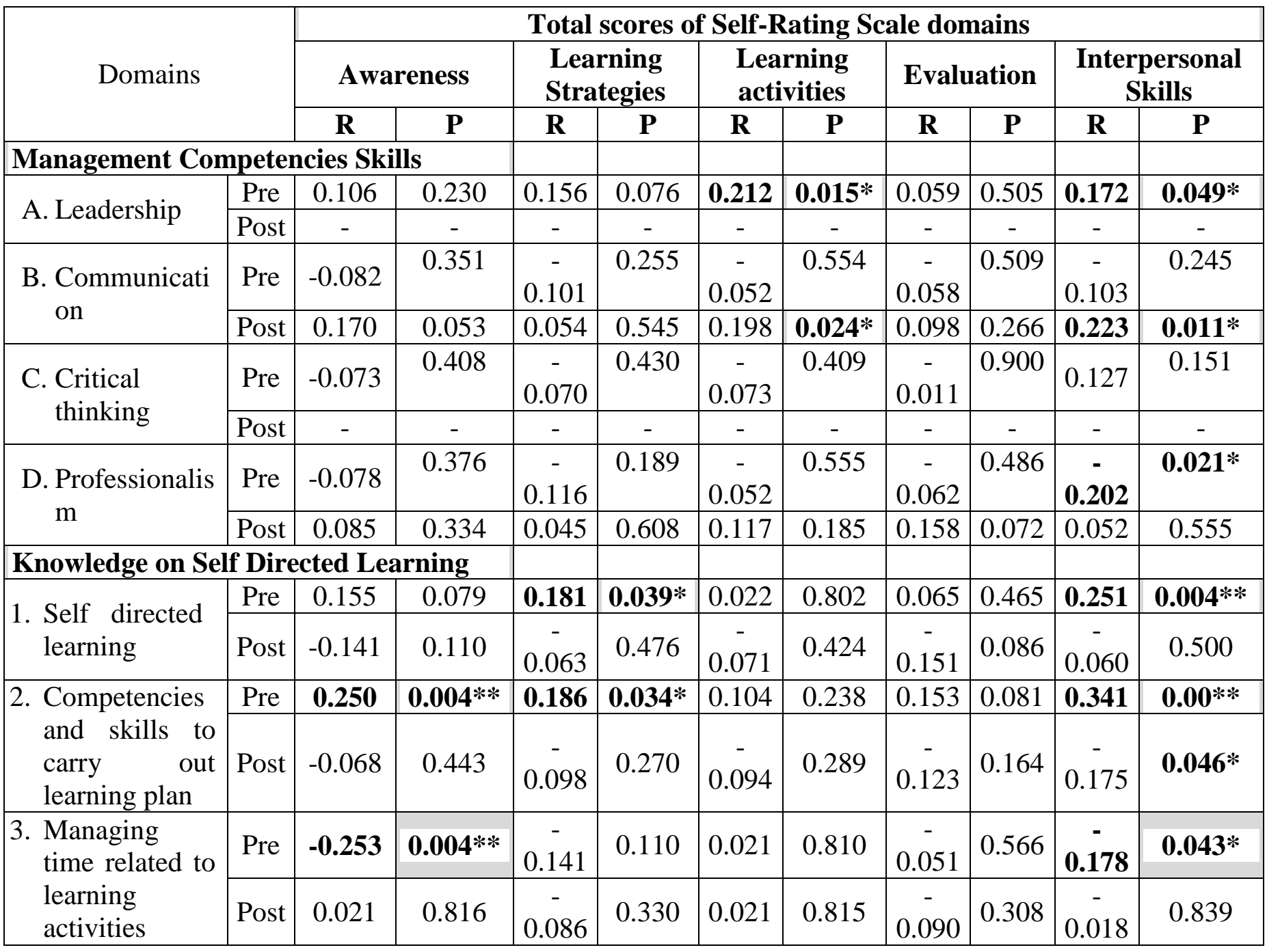

* Significant at $\mathrm{P}<0.05$

** Highly significant at $\mathrm{P}<0.01$ 


\section{Tanta Scientific Nursing Journal}

\section{DISCUSSION}

Technical nursing students' showed internal motivation and tendency to become self directed in their learning, due to being exposed to a diversity of life experiences. Other important factor is their successful completion of undergraduate studies which suggest a satisfactory sense of self-esteem affect positively their motivation for lifelong achievement. So they have high awareness, learning strategies, learning activities, evaluation and interpersonal skills of self directed learning. Jiano $(2015)^{(10)}$ mentioned that undergraduates demonstrate high level of professionalism, independency in learning and leadership in their chosen field. Additionally Zachariah and Huynh (2011) (11) reported that learners are ready and stimulated to learn when they encounter a gap in their current and desired knowledge indicating a high readiness for self-directed learning among the respondent.

All technical nursing students subjects showed weak levels of management skills competencies pre program this result indicates the importance for those technical nursing students to attend training program on self directed learning and management competencies skills. That educational program can assist them on how to develop management competencies and to have ability in their profession, being good communicator and leader as well as being critical thinker. However the competent nursing practice could be promoted through continuous training and developing experiences, regardless of the past education or the graduation level as asserted by Griot $(2016)^{(12)}$.

Finding of the present study is supported by study of Zakaria (2013) ${ }^{(13)}$ found that educational program helped nurses in keeping up to data with new concept, increasing competencies, modifying their attitudes and developing their abilities to deal with patients and problems. Also Mohamed (2012) ${ }^{(14)}$ supported the results and revealed that internship programs allow for professional growth in specific fields and developing their capabilities.

Present study illustrate that preprogram all technical nursing student had weak level of knowledge regarding self directed learning, competencies and skills to carry out learning plan, and managing time related to learning activities. The fact is that preprogram students find it difficult to conduct self directed learning, because their teacher's experiences still emphasize a teacher-centered approach, which still focuses on summative assessment and memorizing facts. Student's background 


\section{Tanta Scientific Nursing Journal}

and cultural factors also contribute to those students's decrease of motivation to conduct self directed learning. Apparently those students require more case or problem-based studies, clinical orientations, innovative teaching programs group discussions and tutorials in regular teaching. So to improve their self-directed learning, they need to take responsibility to plan, implement and evaluate their own learning and to work independently to achieve their learning goals. But immediate and 3month post program knowledge of those students significantly improved regarding to self directed learning due to providing them with explicit advance information about aspects and strategies of self directed learning. Also they provided with assistance to improve their self-management skills and to take control over their own learning especially in respect to time, resources and learning strategies.

Result of this study reveals that there are positive correlations between technical nursing students managerial skills and self directed learning knowledge pre-post program. These correlations are highly positive in both immediately post program and three months later. which means that they acquires more knowledge about managerial skills and self directed learning improved their ability to apply these skills in the clinical training area.

Banan et al, (2017) (15) support present study and found statistical significant positive correlation between nursing students knowledge and practice regarding management skills. Also the study done by Ibrahim (2010) ${ }^{(16)}$ supported results of the present study and found positive correlations between nurse interns' knowledge and their practical skills after their management program.

\section{CONCLUSION \&RECOMMENDATION}

Implementation of self directed learning program had led to improve technical nursing students management skills competencies, and self directed learning knowledge. Self direct learning readiness tool impacted on giving them more confidence to work in their respective practice areas, and made a positive difference in their learning behaviour. Actually pre educational program, majority of technical nursing students had weak level regarding self directed learning knowledge and management skill competencies. Proper design and implementation of self directed learningprogram improved their self directedknowledge and management skill competencies significantly post 


\section{Tanta Scientific Nursing Journal}

educational program with correlation between them.

\section{REFERENCES}

1. Yang G. and Jiang X. Self Directed Learning Readiness and Nursing Competency among Undergraduate Nursing Students in Fujian Province of China. International Journal of Nursing Sciences.2014; 3(1):255-259.

2. Ullah, M. and Anwar, MA. Developing competencies for medical librarians in Pakistan. Health Information \& Libraries Journal.2013;30: 59-71.

3. Beverley D. Development and Evaluation of an Integrated Clinical Learning Model to Inform Continuing Education for Acute Care Nurses. Published Doctoral Thesis.Flinders University.2010:80.

4. Lazarte F.C. Core Competencies of Beginning Staff Nurses: A Basis for Staff Development Training Program. Journal of Advanced Management Science. 2016;4(2).

5. Brown B. From Novice to Expert to Mentor Shaping the Future.American Journal of Critical Care.2011; 13:300-308.

6. Romeo Luis A., Macabasag., Kathleen Kirby. ALeadership, Management and Team Competencies of Filipino Nursing Student Manager-
Leaders: Implications on Nursing EducationInternational Journal of Nursing Science .2016; 6(5): 109-116.

7. Knowles J. and Pickering J. Testing Nursing Staff Competencies Using an Online Education Module. Renal Society of Australasia JournaL. 2012; 8(1): 32-37.

8. Kiessling C., Dieterich A., and Fabry G. et al. Communication and social competencies in medical education in German-speaking countries: the Basel consensus statement. Results of a Delphi survey. Patient Educ Couns. 2010;81(2):259-266.

9. Conner T. The Relationship between Self-Directed Learning and Information Literacy among Adult Learners in Higher Education. Published Doctrol Thesis.Tennesse University .2012:130-132.

10. Jiano L. Practical strategies to promote self-directed learning in the medical curriculum. International Journal of Self-directed Learning.2015; 11 (1): 1-11.

11. Zachariah R. and Huynh S. The selfrating scale of self-directed learning (SRSSDL): a factor analysis of the Italian version. Nurse Educ Today. 2011; 33(12):1511-1516.

12. Griot E. Systematic review of instruments for measuring nurses' 


\section{Tanta Scientific Nursing Journal}

knowledge, skills and attitudes for evidence-based practice. J AdvNurs . 2016; 70(10):2181-2195.

13. Zakaria Z. Quality of Clinical Nursing Round among Nursing Demonstrators and Student. Master Degree. University of Tanta.Faculty of Nursing.Unpublished.2013; 1.

14. Mohammed N. Effect of Educational Transformational Program on Teaching Skills of Nurse Teachers. Doctoral thesis.Ene shams University. Faculty of nursing.Unpublished thesis.2012; 100- 102.

15. Ibrahim L. Impact of management program on competency of graduate students, Tanta University, Doctoral Thesis. 2010.

16. Banan S. and Elsharkawy N. Undergraduate Nursing Students' and Clinical Instructors' Perceptions of the Characteristics of an Effective Clinical Instructor at the Faculty of Nursing, Cairo University American Journal of Nursing Science .2017; 6(3): 185-192. 